\title{
High resolution radar for automobile applications
}

\author{
R. Schneider and J. Wenger \\ DaimlerChrysler AG, Research \& Technology, REM/CR, Wilhelm-Runge-Str. 11, D-89081 Ulm, Germany
}

\begin{abstract}
This contribution points out the potential of radar sensors with high resolution in azimuth and range in traffic environment and addresses some aspects of their practical implementation with respect to system design, hardware and software. A prototypical vehicle-based instrumentation radar is presented, which performs on-line acquisition and real-time visualisation of radar images with an update rate of up to $10 \mathrm{~Hz}$. The enhanced perception capabilities of high resolution radar in traffic environment will be demonstrated with respect to new comfort and safety features, and practical realisation approaches concerning data processing and hardware including the scanning antenna will be discussed.
\end{abstract}

\section{Motivation of high resolution radar for automotive application}

\subsection{Current status}

Millimetre-wave radar has proven to be an ideal sensor in traffic environment (Wenger et al., 2000). At present some car manufacturers offer radar-based autonomous cruise control (ACC) for highway operation as an extra or will do this in the near future. Further development activities of the suppliers are primarily focused on reducing costs and size of the sensor. Although announced on their roadmaps, no additional functionality beyond ACC, like stop-and-go support, obstacle detection, and collision avoidance seems to be in sight for mass products, as this would require clearly enhanced sensor capabilities. Due to the moderate resolution of the employed sensors, insufficient information about the environment is delivered in order to enable advanced driver assistance functions like reliable obstacle warning or emergency breaking.

Conventional ACC radar sensors deliver a target list with distance, azimuth angular position, relative speed, and re-

Correspondence to: R. Schneider

(robert.r.schneider@daimlerchrysler.com) flectivity of significant objects, which typically are smaller than the spatial resolution of the radar. Hence a classification of these point-targets generally is not possible. Table 1 lists some characteristics of an ACC radar sensor (Distronic by Mercedes-Benz). Especially the handling of stationary targets is difficult, as they cannot be separated by differences in their Doppler-shift.

1.2 Specific properties and potential of millimetre-wave radar imaging

The missing capability of object classification and scenario interpretation in first generation ACC sensors could be overcome using a multi- or scanning-beam radar with high resolution in range and azimuth generating real-aperture radar images containing considerably more information about the ambient traffic situation. At the DaimlerChrysler Research Center in Ulm the fundamental potential of high resolution $\mathrm{mm}$-wave radar with respect to automotive applications has been investigated since several years (Schneider et al., 1996, 1997; Schneider and Wenger, 1999). One of the most important results was that vehicles appear as laterally and longitudinally extended objects in high resolution radar images range versus azimuth. Caused by reflections from the road surface, the complete bottom sides can be seen, allowing for the recognition of vehicles and distinction of different classes (cars, vans, trucks) (Schneider et al., 2000). Even obscured vehicles become visible, which provides very important information for the operation in stop-and-go traffic. Another benefit is that road environment like roadsides and other ground structures (crash barriers, reflection posts, traffic signs, etc.) can clearly be identified. As the essential information about the environment is contained in a radar image with the spatial co-ordinates azimuth and range, no resolution in elevation and no large vertical antenna aperture is required. The corresponding line antenna would be well compatible with the vehicle front design.

A crucial advantage of radar sensing is its direct acquisition of velocity via Doppler processing, which could be taken 
Table 1. System parameters of a typical ACC radar sensor

\begin{tabular}{ll}
\hline frequency & $76-77 \mathrm{GHz}$ \\
radar principle & pulse Doppler \\
number of separate beams & 3 \\
beam width (symmetrical) & $3.4 \mathrm{deg}$ \\
range resolution & $5 \mathrm{~m}$ \\
Doppler resolution & $1 \mathrm{~km} / \mathrm{h}$ \\
system sensitivity & $0 \mathrm{dBsm} @ 150 \mathrm{~m}$ \\
cycle time & $70 \mathrm{msec}$ \\
\hline
\end{tabular}

Table 2. Acquisition parameters for high resolution radar data

\begin{tabular}{ll}
\hline centre frequency & $76.5 \mathrm{GHz}$ \\
field-of-view in azimuth & $\pm 10 \mathrm{deg}$ \\
azimuth beam width & $1.0 \mathrm{deg}$ \\
elevation beam width & $5.0 \mathrm{deg}$ \\
range resolution & $1 \mathrm{~m}$ \\
velocity resolution & $1 \mathrm{~km} / \mathrm{h}$ \\
system sensitivity & $-20 \mathrm{dBsm} @ 150 \mathrm{~m}$ \\
\hline
\end{tabular}

as a third dimension in the radar images. Effective data compression is achieved by reducing the velocity information of an azimuth-range resolution cell just to a single value representing the dominant reflection object in this cell. The resulting information is described in two 2D image matrices of radar reflectivity and Doppler. For visualisation purposes both matrices can be combined into a single image using a pseudo-colour coding of Doppler and radar intensity into hue and saturation, for example.

Figure 1 shows an example of such a kind of radar image and exhibits the specific properties of high resolution radar sensing at millimetre wave radar in traffic environment. The spatial radar data acquired in polar co-ordinate grid has been transformed into a Cartesian PPI (planar position indicator) representation, which delivers a geometrically correct bird's eye view of the scene. The camera for video documentation was located close to the antenna position $60 \mathrm{~cm}$ above ground. The colour coding of relative Doppler shift is: yellow for the same speed, red and green for closing and opening Doppler, respectively. Radar acquisition parameters as listed in Table 2 have been applied. Details about the radar set-up will be discussed in the following sections.

Clearly visible is the left roadside with guard rail, which approaches while driving and appears as a red line. Although the right roadside is merely covered with grass, it can be recognised. The preceding truck with trailer appears yellow due to its zero relative Doppler shift, and as an extended area with true dimensions as a result from the road surface reflections. Even a car in front of the truck becomes visible by the wave duct effect. Its green colour indicates an increasing distance from the truck. Here a very attractive feature becomes evident: In platoon traffic not only the next vehicle can be tracked but also at least one ahead. Perceiving its manoeuvres could improve longitudinal vehicle control considerably.
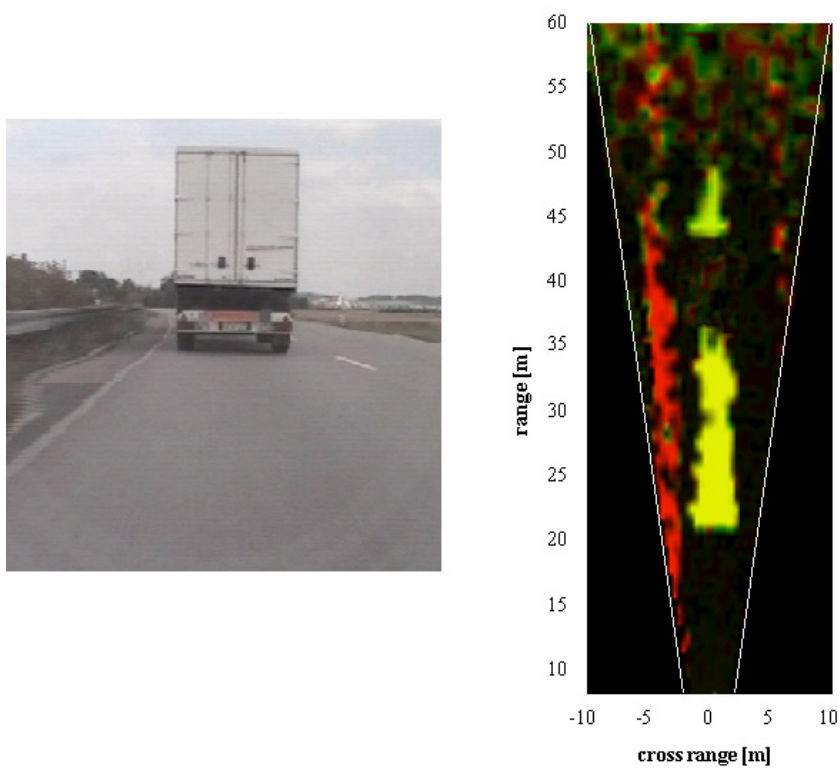

Fig. 1. Traffic scene and corresponding high resolution radar image in PPI representation.

\section{Prototypical realisation of an automotive imaging radar}

Based on the fundamental findings about millimetre-wave imaging an instrumentation radar system for on-line radar image acquisition and real-time visualisation was specified and realised. Although the overall system design is oriented at high performance and versatility, its basic features should have the perspective of mean to long term commercialisation. Installed in a test car the system serves for the detailed evaluation and demonstration of the capabilities of radar imaging, data acquisition, design, and test of processing algorithms, and as a basis for the implementation of new comfort or safety applications. Its operation parameters can be varied in wide ranges in order to achieve different image areas, resolution, and sensitivity.

\subsection{System design}

\subsubsection{Radar parameters}

Imaging of vehicle underbodies and detection of hidden objects via reflections from the road surface require higher sensitivity with respect to a conventional ACC radar sensor. Preliminary investigations resulted in the need of about $20 \mathrm{~dB}$ additional sensitivity in order to enable these features up to $150 \mathrm{~m}$ range, as those targets are characterised by radar cross section (RCS) values of down to $-20 \mathrm{dBsm}$. An increased target dynamic of $50 \mathrm{~dB}$ has to be assumed. Together with the dynamic due to propagation attenuation in $5 . .150 \mathrm{~m}$ range of $60 \mathrm{~dB}$ an overall requirement for system dynamic results, with certainly cannot be handled by a FMCW system. Hence pulsed waveform has been chosen for the imaging radar system design because of its robustness against saturation. 
a)

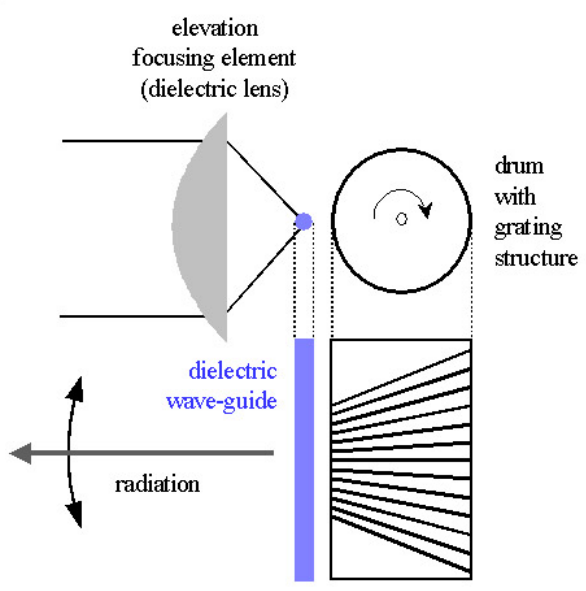

b)

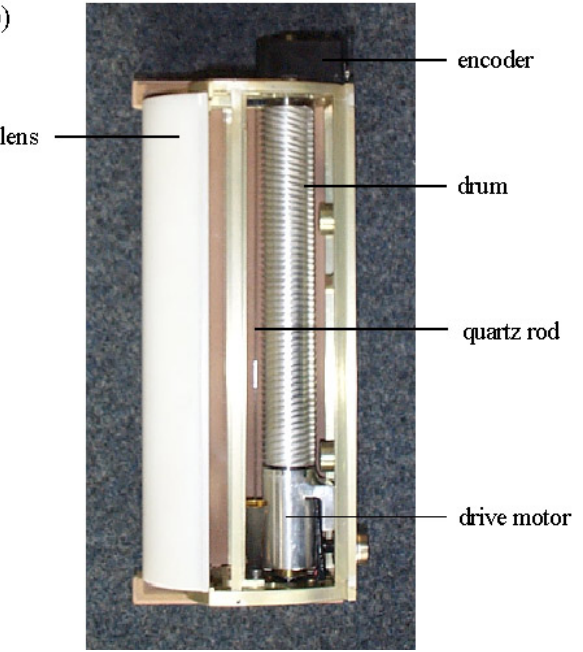

Fig. 2. Schematic diagram (a) and implementation (b) of azimuth scanning fan beam antenna.

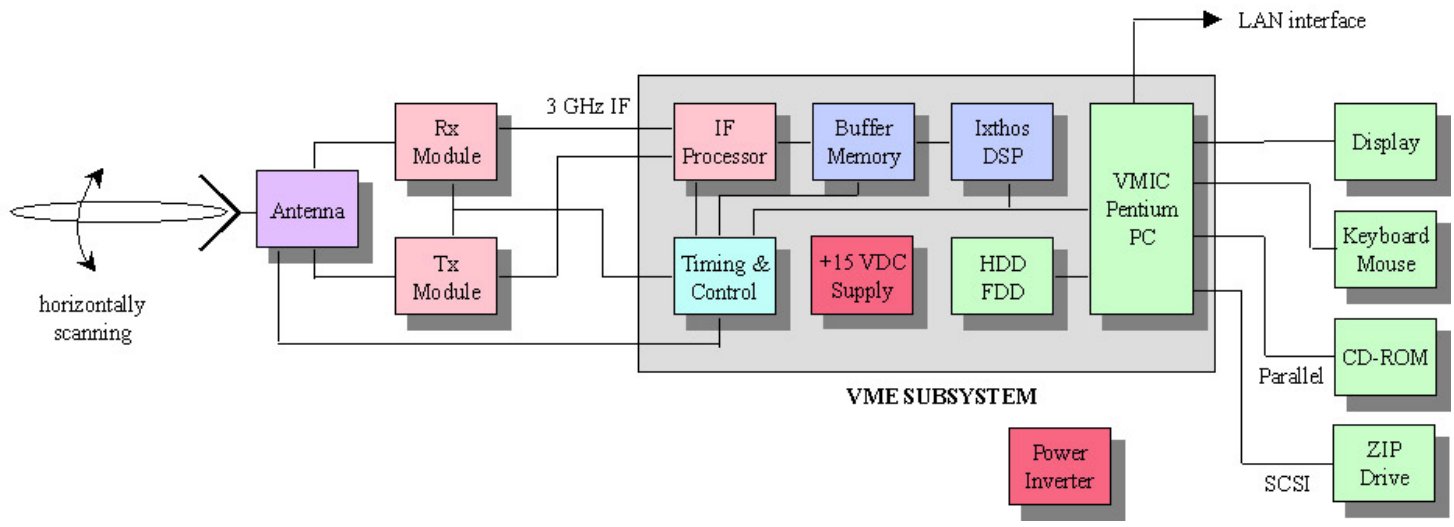

Fig. 3. Block diagram of the complete instrumentation radar system.

The radar transceiver lay-out has been based on practical imaging parameters (see Table 2). Range resolution of $1 \mathrm{~m}$ corresponds to $7 \mathrm{nsec}$ pulse duration and to $150 \mathrm{MHz}$ bandwidth of a matched IF filter. The gain of an antenna with beam widths of $1 \mathrm{deg} \times 5 \mathrm{deg}$ is assumed to be at least $38 \mathrm{dBi}$, which contributes considerably to the additionally required sensitivity. Speed resolution in the Doppler processing depends on the number of range bins $\mathrm{N}$ and the pulse repetition frequency PRF. A practical choice is $\mathrm{N}=512$ and $\mathrm{PRF}=70 \mathrm{kHz}$, which provides sufficient unambiguous speed $(500 \mathrm{~km} / \mathrm{h})$, unambiguous range $(2 \mathrm{~km})$, and processing gain $(27 \mathrm{~dB})$.

A minimum signal-to-noise ratio of $10 \mathrm{~dB}$ should be present in the radar images in order to achieve acceptable detection and false alarm rates. The overall system losses of radome, front-end, and signal processing are estimated not to exceed $8 \mathrm{~dB}$. The transceiver design finally has to find a trade-off between transmit power and receiver noise figure $\mathrm{F}$. Assuming a value of $\mathrm{F}=8 \mathrm{~dB}$ achievable with state-of-theart balanced Schottky mixers, a transmit power of $19 \mathrm{dBm}$ $(80 \mathrm{~mW})$ would be necessary. As this hardly can be realised with today's MMIC technology, a low-noise receive amplifier (LNA) with a noise figure of $4.5 \mathrm{~dB}$ was provided to reduce the required transmit (peak) power to a practical value of about $35 \mathrm{~mW}$. The resulting mean transmit power is below $20 \mu \mathrm{W}$.

The front-end design should demonstrate a long term producibility potential (cost aspect), which won't allow for parallel transceiver concepts. This means that the complete field-of-view (FOW) has to be scanned sequentially with a single antenna beam, provided that time-on-target requirements can be satisfied. The above-mentioned Doppler acquisition of 512 samples with a PRF of $70 \mathrm{kHz}$ delivers full integration gain for a minimum time-on-target of about $7 \mathrm{msec}$. The required FOW is deduced from the demand of operation in urban area or narrow road curvature. Case studies show that a coverage of \pm 15 deg is fully sufficient, $\pm 10 \mathrm{deg}$ is acceptable with restrictions in urban (crossing) environment. Assuming an azimuth beam-width of $1 \mathrm{deg}$, the maximum achievable image update rate would be $(7 \mathrm{msec} \cdot 30)^{-1}$ $=4.8 \mathrm{~Hz}$ in the first and $7.1 \mathrm{~Hz}$ in the second case. Compared to a video system the update rate of an imaging radar 

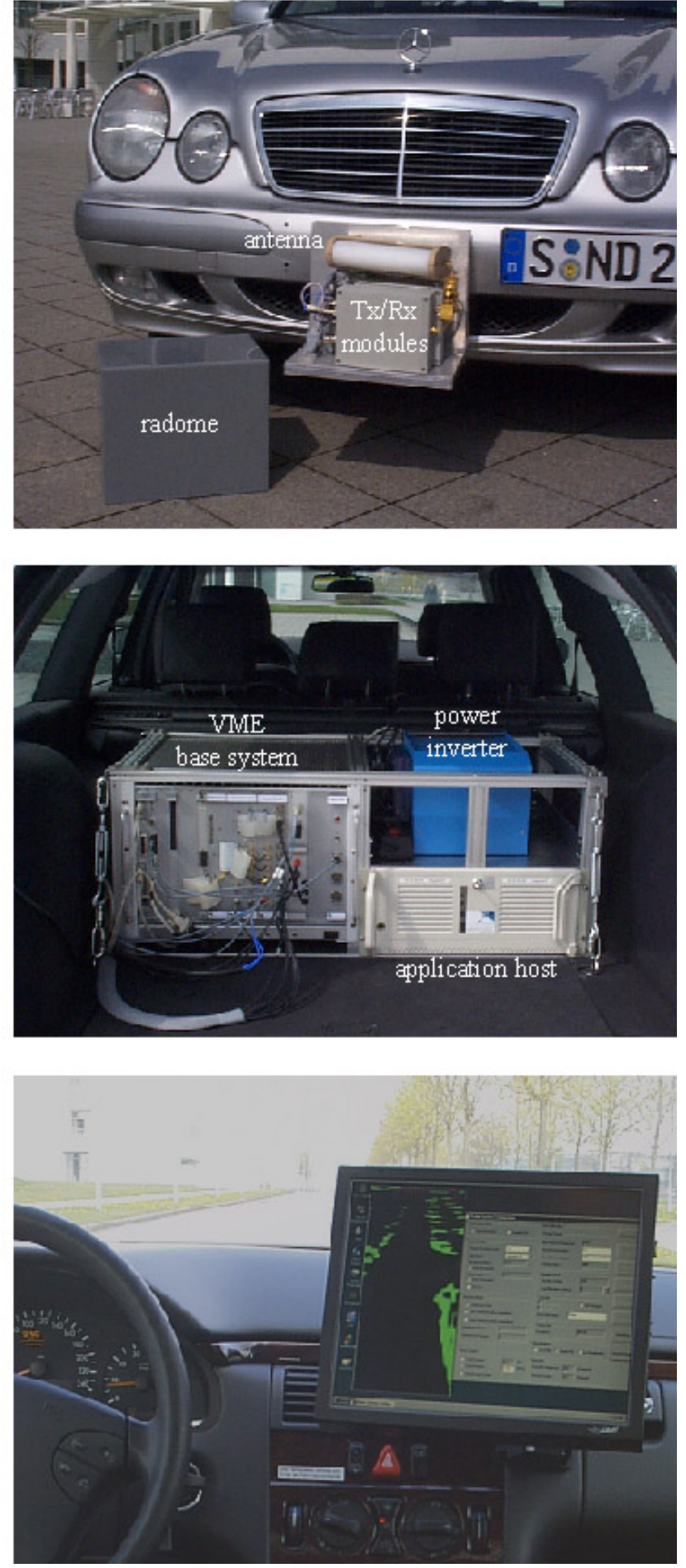

Fig. 4. Test car equipped with imaging radar: (a) front-end, (b) base system, (c) operation and visualisation display.

is allowed to be clearly lower, as the velocity information is directly available and intermediate images can be computed by extrapolation. Hence the mentioned update rates are assumed to be sufficient.
Table 3. Antenna characteristics

$\begin{array}{ll}\text { frequency } & 76-77 \mathrm{GHz} \\ \text { field-of-view in azimuth } & \pm 11.5 \mathrm{deg} \\ \text { azimuth beam width } & 1.5 \mathrm{deg} \\ \text { elevation beam width } & 4.2 \mathrm{deg} \\ \text { gain } & \geq 35.5 \mathrm{dBi} \\ \text { side-lobe suppression } & \leq-15 \mathrm{~dB} \\ \text { maximum scan rate } & 20 \mathrm{~Hz} \\ \text { dimensions (width } \times \text { height } \times \text { depth) } & 24 \mathrm{~cm} \times 6 \mathrm{~cm} \times 10 \mathrm{~cm}\end{array}$

\subsubsection{High resolution azimuth scanning antenna}

The antenna forms the actual technological key component in a real aperture imaging radar. The challenge is to combine fast beam scanning of a wide FOW at good electrical properties (efficiency, side-lobes) with the potential of low-cost production, compactness, and mechanical robustness. Phased array concepts fail due to their complexity, focal plane concepts have shortcomings in achieving large FOVs, beam switching leads to high insertion loss in the required switch cascade, and pure mechanical scan isn't practicable for the large aperture.

A very attractive approach, which doesn't completely dispense with moving parts, is a tricky advancement of the image line antenna (Solbach and Wolff, 1985), which exploits the fact that inhomogeneities close to a dielectric wave-guide will cause radiation. An equidistant structure (grating) parallel to the wave-guide effects the radiation of a wave-front with a direction depending on the grating period. If now gratings with varying spacing are arranged on the circumference of a drum (see Fig. 2a), sawtooth-wise scanning of the radiated wave-front is achieved by acceleration-free rotation of the drum (Manasson et al., 1996). For beam forming in the orthogonal (elevation) plane either a cylindrical parabolic reflector or dielectric lens can be used.

Figure $2 \mathrm{~b}$ shows the prototypic implementation of such a "spinning grating" antenna with lens (design and manufacturing by WaveBand Corp., USA), which was applied for the instrumentation radar set-up. The grating structure is realised by grooves with different pitch in a metallic cylinder resulting in a continuous beam scanning behaviour. A rigid quartz rod of about $1 \mathrm{~mm}$ diameter forms the dielectric wave-guide. A mode launcher allows the antenna to be connected via a standard WR-12 wave-guide flange. The drum driving is performed by a small DC gear motor (which could be integrated into the drum to increase compactness), an angular encoder serves for rotation speed control and exact reference for the beam pointing direction.

Practical experience with the antenna proves well applicability in harsh automotive environment. Some electrical and mechanical characteristics are compiled in Table 3 (verified by measurements). The azimuth beam-width is somewhat larger and accordingly the gain lower than postulated in above system design, but for availability reasons this had to be accepted. 


\subsection{Description of the hardware and vehicle installation}

The instrumentation radar is designed as a versatile test system with real-time processing and visualisation features, which requires an open and universal hardware structure. It consists of a base unit in a standard VME chassis and the remote front-end (transmitter and receiver modules plus antenna). Figure 3 depicts the overall block diagram. The central component is an embedded Pentium III PC with Windows NT performing system controlling, radar image composition and pre-processing, visualisation, and data handling. Its main periphery is formed by timing and control electronics generating all the radar timing and a number of direct control signals, an IF processor module for converting the IF signal from the receiver ( $\mathrm{Rx})$ module into base-band I/Q signals and digitisation, a digital signal processor (DSP) performing the Doppler FFTs and other numerically expensive pre- processing (details see below), and a number of common devices.

System operation is done via a graphical user interface (GUI) allowing the adjustment of pertinent radar parameters like transmit pulse width, PRF, IF filter bandwidth, range sampling, number of Doppler bins, and receiver gain.

A ferrite circulator for transmit and receive duplex is mounted together with a receive LNA directly at the antenna and connects to the Tx and Rx modules. The transmit signal is generated in a phase locked dielectric resonator oscillator (DRO) at $19.125 \mathrm{GHz}$, pulse gated in a two-stage PINmodulator, and multiplied to $76.5 \mathrm{GHz}$ in an active quadrupler with an output power of about $16 \mathrm{dBm}$. The minimum pulse length is $3.5 \mathrm{nsec}$. Key component in the Rx module is a biased, balanced fundamental mixer (measured noise figure $\mathrm{F}=7.6 \mathrm{~dB}$ ). It converts to an IF signal of $3 \mathrm{GHz}$, which is led to the base system on a high power level in order to minimise interference in the long transmission line.

The detailed design and implementation of the VME base system and the Tx/Rx modules as well as programming the control software of the VME components were performed by Innovative Technology, USA.

Figure 4 shows the integration of the system into a test car. The front-end components are mounted centred at the front bumper in a weather-proof housing (Fig. 4a). The antenna height is positioned $60 \mathrm{~cm}$ above ground, which is a good compromise between exploiting multi-path effects by road surface reflections (hidden cars, vehicle underbodies) and roadside detection. Also with respect to shadowing effects of road crests (Schneider et al., 2000) the antenna position shouldn't be to low. The radome is made of $5 \mathrm{~mm}$ polypropylene (according to the multiple half-wavelength matching condition) and showed less than $0.2 \mathrm{~dB}$ two-way insertion loss.

The base system is rack-mounted together with its $\mathrm{AC}$ $230 \mathrm{~V}$ power supply in the trunk of the estate car (see Fig. 4b). An additional component placed there is a second PC provided for the intended implementation of safety or comfort applications. In the current status of the set-up it serves for synchronised video documentation and is connected to the radar control PC via Ethernet LAN.

For GUI operation of the system and the on-line radar image visualisation a display is attached at the dashboard (Fig. 4c). Note the junction on the left hand side recognisable in the instantaneous radar image.

\subsection{Data processing}

The imaging radar data processing is performed in the following subsequent stages: radar data pre-processing, composition of radar images, image pre-processing, and conditioning for visualisation. Most of the pre-processing is performed in the DSP card, primarily computation of the Doppler FFTs for each resolution cell and maximum search in the Doppler frequency spectra. The resulting range profiles of reflectivity and associated Doppler values have to be assembled into the actual 2D radar images. As radar acquisition is not synchronised with the antenna pointing direction, this is done based on the encoder values and interpolating onto a fix azimuth grid. The antenna scan speed is controlled in a feed-back loop including the complete processing chain, so that the azimuth increment between subsequent range profile measurements is exactly the $3 \mathrm{~dB}$ beam width (Nyquist condition). With the currently used antenna this results in 16 azimuth directions per scan. Range is typically sampled from $5 \mathrm{~m}$ to $150 \mathrm{~m}$ at $1 \mathrm{~m}$ resolution, resulting in raw radar images of $16 \times 145$ pixels. Range dependent amplitude correction is performed by $R^{p}$ with an exponent $p \approx 1$ as a good compromise between point target detectability and noise limitation at larger ranges.

Some image pre-processing is performed in order to reduce artefacts caused by system deficiencies or physical effects. Cross-coupling in the transceiver or antenna leads to near range echoes at zero Doppler. They are blanked by a kind of adaptive MTI filter. Because of the relatively low side-lobe suppression of the antenna cross-range blooming of strongly reflecting objects occurs. It is reduced by appropriate lowering the level of those pixels having the same range and Doppler as this object. Another physical effect occurring in the presence of strong reflections is double bounce resulting in false echoes at twice range and twice Doppler in the images. They can be eliminated by pixel-wise checking for this condition.

In order to achieve a proper mapping of intensity values into indices suited for an image colour coding, low and high level clipping has to be performed on the logarithmic values. A heuristically optimised choice is: low level clipping at $10 \mathrm{~dB}$ above noise floor (this is the above specified SNR) and establishing a dynamic head room of $20 \mathrm{~dB}$. The noise floor can be computed directly from the measured data by averaging the intensity of target-free resolution cells, which allows a brightness control of the radar images independent of any system parameters.

For visualisation conditioning 2D interpolation of the radar data has to be performed, which favourably is done directly in the desired co-ordinate system, either Cartesian 
(PPI plot) or vanishing point projection with direct relation to optical vision. Due to the moderate FOV, trigonometric approximations can be applied in the transform formulas, which minimise the computational effort. Final step is combining intensity and Doppler matrices into a single one for pseudo-colour coding of a specific colour-map.

All of the described data processing could be implemented on the base-system PC controller without significant reduction of real-time capability. With the above mentioned radar and imaging parameters an update rate of $3 \mathrm{~Hz}$ results, by reduced range and Doppler settings it can be increased to $10 \mathrm{~Hz}$. Additionally to visualisation the acquired data can be recorded either in MATLAB binary file or TIF image format for off-line processing.

\subsection{Imaging performance}

The sensitivity of the mobile instrumentation radar could be verified as predicted in the system design. Hidden vehicles proved to be detectable in all relevant situations. Roadsides can typically be recognised up to a range of $70 \mathrm{~m}$, provided they consist of gravel, grass, or other vegetation (road curvature prediction based on real clothoid parameter modelling and tracking in consecutive images will work clearly farther). Radar echoes from the underbodies reliably appear in two-dimensional representation with correct dimensions and alignment in the radar images and encourage the development of corresponding classification algorithms for traffic scene interpretation.

Spurious objects are to be found regularly in the presence of guard rails, metallic fences, or kerbstones parallel to the road. Due to detour propagation vehicles appear virtually mirrored on the other side of these reflecting areas. The forward reflectivity even of low kerbstones is very high, explicable by their dihedral appearance. But here an exploitable effect becomes evident: Although nearly no backscatter can be observed from kerbstones, occurrence of multipath reflections of preceding vehicles allows localising them in the middle between direct and virtual image. This expands the capability of roadside recognition.

\section{Applications}

Some indications have been made in the above sections concerning new applications for an automotive high resolution radar with imaging capabilities. Currently it is presumed that the following functionalities can be achieved or at least supported by such an environmental sensor:

- improvement of ACC function (higher speed, farther range, use also off highways)

- obstacle warning (including stationary objects - for example rear end of traffic jam)

- emergency breaking

- collision avoidance
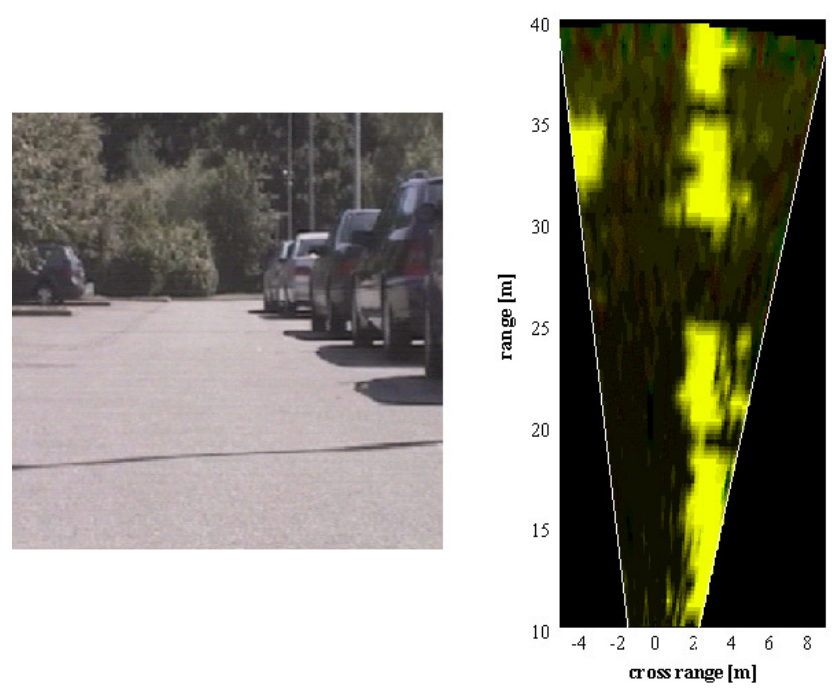

Fig. 5. Imaging capability of free parking space identification.

- stop-and-go traffic support

- detection of pedestrians

- identification of parking space

- curve warning

- visibility enhancement

Some of these are not stand-alone applications, but have to be seen in combination with others. Parking space identification would be an interesting enhancement for automatic parking systems, which currently are under development. The sensing capability of high resolution radar has been investigated by measurements in according situations and is illustrated in Fig. 5. A free parking space can be identified clearly earlier than by visual inspection and a rough size determination is possible (the free space in the analysed scene was $5 \mathrm{~m} \mathrm{long}$ ).

With respect to safety applications pedestrian detection also can't be an autonomous feature, as the spatial resolution of the imaging radar won't allow classification of persons. But localisation of potentially relevant objects would be a very important input for sensor fusion approaches. Radar can identify regions of interest (ROIs), in which detailed analysis based on video image processing, e.g. is performed in Heisele et al. (1996). This provides also the information redundancy, which is required in safety systems, as radar and video sensing is largely complementary.

A specific contribution of radar can be the detection of pedestrians hidden by vehicles, for example at a bus stop. Here again the wave duct effect between road surface and vehicle underbody becomes beneficial. This could help to recognise situations of persons abruptly entering the roadway (playing children). Radar measurements have been performed indicating that persons can be detected reliably in front of a bus, provided the distance is greater than the range resolution ( $1 \mathrm{~m}$ in the current system realisation). 

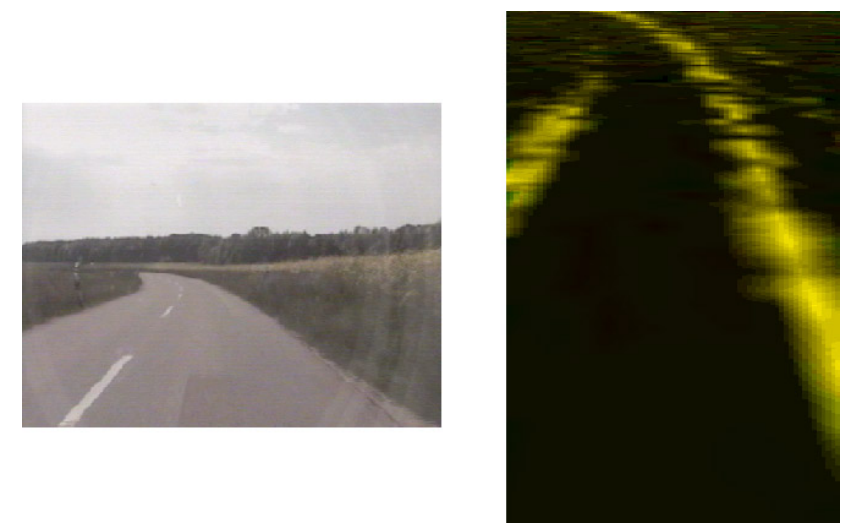

Fig. 6. Roadside detection capability.

An interesting application based solely on radar information could be a kind of "curve assistent", which warns the driver in the danger of overshooting a curve. Exploiting the capability of continuous roadside detection even in the absence of any border structures, the road course and its curvature can be determined and related to the actual speed, which is directly available from the Doppler data. Figure 6 gives an impression of the roadside detection performance in the situation of a narrow country road. Maximum acquisition range has been $100 \mathrm{~m}$. In this example a vanishing point projection has been applied to the radar image.

\section{Conclusion and outlook}

It could be shown that by moderate increasing of resolution, angular coverage, and sensitivity with respect to existing automotive radar sensors a new quality of environmental sensing can be established. It enables a fundamentally new approach of radar data interpretation: image processing based scenario analysis instead of point target tracking. This opens up new perspectives of comfort and safety features in future automobiles. The practical implementation of a prototypic mobile imaging radar proves technical feasibility at system design parameters, which are not contradicting to commercialisation aspects like complexity, size, and cost.
Future activities now will be hardware- and softwarerelated as well. Mass production realisation aspects of the front-end (antenna and millimetre-wave electronics) have to be clarified. This includes also evaluation of upcoming new technologies. Autonomous implementation of the above mentioned comfort or safety features requires the development of radar image processing algorithms (based on the adaptation of established methods like edge detection, segmentation, clustering, tracking, object classification) and scene interpretation according to the particular application. An important aspect will also be fusion of radar and vision data, for which an optimised concept has to be worked out.

\section{References}

Wenger, J., Schneider, R., Stotz, M., Danner, B., Lauer, W., Nieuwenhuizen, R., and Adomat, R.: Forward Looking Radar Activities at DaimlerChrysler; Workshop on Automotive Millimetre-Wave Radar Systems (WS 3), EuMC 2000, Paris, France, pp. I,1-I,8, 3 October 2000.

Schneider, R., Wanielik, G., and Wenger, J.: Millimeterwave imaging of traffic scenarios; Intelligent Vehicles '96, Tokyo, Japan, Sept. 18-20, pp. 327-332, 1996.

Schneider, R., Wenger, J., Wanielik, G., and Neef, H.: Millimeterwave images of traffic scenes and their automatic interpretation; Conf. Proc. Microwaves and RF, London, UK, 30 Sept.-2 Oct., pp. 239-244, 1997.

Schneider, R. and Wenger, J.: System Aspects for Future Automotive Radar; Proc. of the IEEE MTT-S Microwave Symposium, 1999.

Schneider, R., Didascalou, D., and Wiesbeck, W.: Impact of road surfaces on millimeter wave propagation; IEEE Trans. Veh. Tech. Vol. 49, No.4, pp. 1314-1320, July 2000.

Solbach, K. and Wolff, I.: Dielectric Image Line Groove Antennas for Millimeter Waves, Part II: Experimental Verification; IEEE Trans. AP, vol. AP-33, No. 7, pp. 697-706, July 1985.

Manasson, V., Sadovnik, L., and Mino, R.: MMW Scanning Antenna; IEEE AES Systems Magazine, pp. 29-33, October 1996.

Heisele, B., Neef, N., Ritter, W., Schneider, R., and Wanielik, G.: Object Detection in Traffic Scenes by a Colour Video and Radar Data Fusion Approach; First Australian Data Fusion Symposium ADF-96, Adelaide, Australia, pp. 48-52, November 1996. 